\title{
TRUNCATED TOEPLITZ OPERATORS: SPATIAL ISOMORPHISM, UNITARY EQUIVALENCE, AND SIMILARITY
}

\author{
JOSEPH A. CIMA, STEPHAN RAMON GARCIA, WILLIAM T. ROSS, \\ AND WARREN R. WOGEN
}

\begin{abstract}
A truncated Toeplitz operator $A_{\varphi}: \mathcal{K}_{\Theta} \rightarrow \mathcal{K}_{\Theta}$ is the compression of a Toeplitz operator $T_{\varphi}: H^{2} \rightarrow H^{2}$ to a model space $\mathcal{K}_{\Theta}:=H^{2} \ominus \Theta H^{2}$. For $\Theta$ inner, let $\mathcal{T}_{\Theta}$ denote the set of all bounded truncated Toeplitz operators on $\mathcal{K}_{\Theta}$. Our main result is a necessary and sufficient condition on inner functions $\Theta_{1}$ and $\Theta_{2}$ which guarantees that $\mathcal{T}_{\Theta_{1}}$ and $\mathcal{T}_{\Theta_{2}}$ are spatially isomorphic. (i.e., $U \mathcal{T}_{\Theta_{1}}=\mathcal{T}_{\Theta_{2}} U$ for some unitary $U: \mathcal{K}_{\Theta_{1}} \rightarrow \mathcal{K}_{\Theta_{2}}$ ). We also study operators which are unitarily equivalent to truncated Toeplitz operators and we prove that every operator on a finite dimensional Hilbert space is similar to a truncated Toeplitz operator.
\end{abstract}

\section{INTRODUCTION}

In this paper we consider several questions concerning spatial isomorphism, unitary equivalence, and similarity in the setting of truncated Toeplitz operators. Loosely put, a truncated Toeplitz operator is the compression $A_{\varphi}: \mathcal{K}_{\Theta} \rightarrow \mathcal{K}_{\Theta}$ of a standard Toeplitz operator $T_{\varphi}: H^{2} \rightarrow H^{2}$ to a Jordan model space $\mathcal{K}_{\Theta}:=$ $H^{2} \ominus \Theta H^{2}$ (here $\Theta$ denotes an inner function). We discuss these definitions and the related preliminaries in Section 2. The reader is directed to the recent survey of Sarason 23] for a more thorough account.

For a given inner function $\Theta$, we let $\mathcal{T}_{\Theta}$ denote the set of all bounded truncated Toeplitz operators on $\mathcal{K}_{\Theta}$. The main result of the paper (Theorem 3.3) is a simple necessary and sufficient condition on inner functions $\Theta_{1}$ and $\Theta_{2}$ which guarantees that the corresponding spaces $\mathcal{T}_{\Theta_{1}}$ and $\mathcal{T}_{\Theta_{2}}$ are spatially isomorphic (i.e., $U \mathcal{T}_{\Theta_{1}}=$ $\mathcal{T}_{\Theta_{2}} U$ for some unitary $\left.U: \mathcal{K}_{\Theta_{1}} \rightarrow \mathcal{K}_{\Theta_{2}}\right)$. This result and its ramifications are discussed in Section 3 while the proof is presented in Section 4 .

In Section 5 , we study the operators which are unitarily equivalent to truncated Toeplitz operators (UETTO). The class of such operators is surprisingly large and includes, for instance, the Volterra integration operator 21. We add to this class by showing that several familiar classes of operators (e.g., normal operators) are UETTO.

We conclude this paper in Section 6 by showing that every operator on a finite dimensional Hilbert space is similar to a truncated Toeplitz operator (Theorem 6.1). In other words, we prove that the inverse Jordan structure problem is always solvable in the class of truncated Toeplitz operators. This stands in contrast to the situation for Toeplitz matrices [15]. 


\section{Preliminaries}

In the following, $H^{2}$ denotes the classical Hardy space on the open unit disk $\mathbb{D}$ [9, 13. The unit circle $|z|=1$ is denoted by $\partial \mathbb{D}$ and we let $L^{2}:=L^{2}(\partial \mathbb{D})$ and $L^{\infty}:=L^{\infty}(\partial \mathbb{D})$ denote the usual Lebesgue spaces on $\partial \mathbb{D}$.

Model spaces. To each non-constant inner function $\Theta$ there corresponds a model space $\mathcal{K}_{\Theta}$ defined by

$$
\mathcal{K}_{\Theta}:=H^{2} \ominus \Theta H^{2}
$$

This terminology stems from the important role that $\mathcal{K}_{\Theta}$ plays in the model theory for Hilbert space contractions - see [18, Part C].

The kernel functions

$$
K_{\lambda}(z)=\frac{1-\overline{\Theta(\lambda)} \Theta(z)}{1-\bar{\lambda} z}, \quad z, \lambda \in \mathbb{D},
$$

belong to $\mathcal{K}_{\Theta}$ and enjoy the reproducing property

$$
\left\langle f, K_{\lambda}\right\rangle=f(\lambda), \quad \lambda \in \mathbb{D}, f \in \mathcal{K}_{\Theta} .
$$

If $\Theta$ has an angular derivative in the sense of Carathéodory (ADC) at $\lambda \in \partial \mathbb{D}$ [23. Sect. 2.2] then $K_{\lambda}$ belongs to $\mathcal{K}_{\Theta}$ and the formulae (2.2) and (2.3) still hold. Letting $P_{\Theta}$ denote the orthogonal projection of $L^{2}$ onto $\mathcal{K}_{\Theta}$, we observe that

$$
\left[P_{\Theta} f\right](\lambda)=\left\langle f, K_{\lambda}\right\rangle, \quad f \in L^{2}, \lambda \in \mathbb{D} .
$$

The preceding formula remains valid for $\lambda \in \partial \mathbb{D}$ so long as $\Theta$ has an ADC there.

We let

$$
k_{\lambda}:=\frac{K_{\lambda}}{\left\|K_{\lambda}\right\|}
$$

denote the normalized reproducing kernel at $\lambda$ and, when we wish to be specific about the underlying inner function $\Theta$ involved, we write $K_{\lambda}^{\Theta}$ and $k_{\lambda}^{\Theta}$ in place of $K_{\lambda}$ and $k_{\lambda}$, respectively.

There is a natural conjugation (a conjugate-linear isometric involution) on $\mathcal{K}_{\Theta}$ defined in terms of boundary functions by

$$
C f:=\overline{f z} \Theta .
$$

Although at first glance the expression $\overline{f z} \Theta$ in (2.6) does not appear to correspond to the boundary values of an $H^{2}$ function, let alone one in $\mathcal{K}_{\Theta}$, a short computation using (2.1) reveals that if $f \in \mathcal{K}_{\Theta}$ and $h \in H^{2}$, then $\langle C f, \Theta h\rangle=0=\langle C f, \overline{z h}\rangle$ whence $C f$ indeed belongs to $\mathcal{K}_{\Theta}$.

A short calculation reveals that

$$
\left[C K_{\lambda}\right](z)=\frac{\Theta(z)-\Theta(\lambda)}{z-\lambda} .
$$

Moreover, the preceding also holds for $\lambda \in \partial \mathbb{D}$ so long as $\Theta$ has an ADC there.

Truncated Toeplitz operators. Since $\mathcal{K}_{\Theta}$ is the closed linear span of the backward shifts $S^{*} \Theta, S^{* 2} \Theta, \ldots$ of $\Theta$ [4, p. 83], where $S^{*} f=(f-f(0)) / z$, it follows that the subspace

$$
\mathcal{K}_{\Theta}^{\infty}:=\mathcal{K}_{\Theta} \cap H^{\infty}
$$

of all bounded functions in $\mathcal{K}_{\Theta}$ is dense in $\mathcal{K}_{\Theta}$. 
Keeping these results in mind, for a fixed inner function $\Theta$ and any $\varphi \in L^{2}$, the corresponding truncated Toeplitz operator $A_{\varphi}: \mathcal{K}_{\Theta} \rightarrow \mathcal{K}_{\Theta}$ is the densely defined operator

$$
A_{\varphi} f=P_{\Theta}(\varphi f)
$$

When we wish to be specific about the underlying inner function $\Theta$, we use the notation $A_{\varphi}^{\Theta}$ to denote the truncated Toeplitz operator with symbol $\varphi$ acting on the model space $\mathcal{K}_{\Theta}$. In most cases, however, $\Theta$ is clear from context and we simply write $A_{\varphi}$.

Although one can pursue the subject of unbounded truncated Toeplitz operators much further [24, 25], we are concerned here with those which have a bounded extension to $\mathcal{K}_{\Theta}$.

Definition 2.8. Let $\mathcal{T}_{\Theta}$ denote the set of all truncated Toeplitz operators which extend boundedly to all of $\mathcal{K}_{\Theta}$.

Certainly $A_{\varphi} \in \mathcal{T}_{\Theta}$ when $\varphi \in L^{\infty}$. However [23, Thm. 3.1], there are an abundance of unbounded $\varphi \in L^{2}$ for which $A_{\varphi} \in \mathcal{T}_{\Theta}$. It is important to note that $\mathcal{T}_{\Theta}$ is not an algebra since the product of truncated Toeplitz operators need not be a truncated Toeplitz operator (a simple counterexample can easily be deduced from [23. Thm. 5.1]). On the other hand, it turns out that $\mathcal{T}_{\Theta}$ is a weakly closed linear subspace of the bounded operators on $\mathcal{K}_{\Theta}$ [23, Thm. 4.2]. Moreover, if $\Theta$ is a finite Blaschke product of order $n$, then one can show that $\operatorname{dim} \mathcal{T}_{\Theta}=2 n-1$ (see Lemma 2.17 below).

Complex symmetric operators. Of particular importance to the study of truncated Toeplitz operators is the notion of a complex symmetric operator [11, 12. Let us briefly discuss the necessary preliminaries. In the following, we let $\mathcal{H}$ denote a separable complex Hilbert space and $\mathcal{B}(\mathcal{H})$ denote the bounded linear operators on $\mathcal{H}$.

Definition 2.9. A conjugation on $\mathcal{H}$ is a conjugate-linear operator $C: \mathcal{H} \rightarrow \mathcal{H}$, which is both involutive (i.e., $C^{2}=I$ ) and isometric (i.e., $\langle C x, C y\rangle=\langle y, x\rangle$ for all $x, y \in \mathcal{H})$.

The standard example of a conjugation is entry-by-entry complex conjugation on an $l^{2}$-space. In fact, each conjugation is unitarily equivalent to the canonical conjugation on a $l^{2}$-space of the appropriate dimension [11, Lem. 1]. Having discussed conjugations, we next consider certain operators which are compatible with them.

Definition 2.10. We say that $T \in \mathcal{B}(\mathcal{H})$ is $C$-symmetric if $T^{*}=C T C$ for some conjugation $C$ on $\mathcal{H}$. We say that $T$ is complex symmetric if there exists a conjugation $C$ with respect to which $T$ is $C$-symmetric.

Recall the conjugation $C$ defined on $\mathcal{K}_{\Theta}$ from (2.6). The following result is from [11.

Proposition 2.11. Every $A_{\varphi} \in \mathcal{T}_{\Theta}$ is C-symmetric.

Clark operators. Let us now review a few necessary facts about the theory of Clark unitary operators 5 . For a more complete account of this theory we refer the reader to [3, 19, 22. To avoid needless technicalities, we assume that the underlying 
inner function $\Theta$ satisfies $\Theta(0)=0$. For $\alpha \in \partial \mathbb{D}$, the operator $U_{\alpha}: \mathcal{K}_{\Theta} \rightarrow \mathcal{K}_{\Theta}$ defined by the formula

$$
U_{\alpha} f=A_{z} f+\alpha\langle f, \bar{z} \Theta\rangle 1, \quad f \in \mathcal{K}_{\Theta},
$$

is called a Clark operator. One can show that each Clark operator $U_{\alpha}$ is unitary and that every unitary rank-one perturbation of the truncated shift operator $A_{z}$ takes the form $U_{\alpha}$ for some $\alpha \in \partial \mathbb{D}$. Less well-known is the fact that each Clark operator $U_{\alpha}$ on $\mathcal{K}_{\Theta}$ belongs to $\mathcal{T}_{\Theta}$ [23, p. 524]. There is also the following theorem [23, p. 515].

Theorem 2.13 (Sarason). If $A$ is a bounded operator on $\mathcal{K}_{\Theta}$ which commutes with $U_{\alpha}$ for some $\alpha \in \partial \mathbb{D}$, then $A \in \mathcal{T}_{\Theta}$.

Since $U_{\alpha}$ is a cyclic unitary operator [3, Thm. 8.9.10], the Spectral Theorem asserts that there is a measure $\mu_{\alpha}$ on $\partial \mathbb{D}$ such that $U_{\alpha}$ is unitarily equivalent to the operator $\left[M_{\zeta} f\right](\zeta)=\zeta f(\zeta)$ of multiplication by the independent variable $\zeta$ on $L^{2}\left(\mu_{\alpha}\right)$. Moreover, the measure $\mu_{\alpha}$ is carried by the set

$$
E_{\alpha}:=\left\{\zeta \in \partial \mathbb{D}: \lim _{r \rightarrow 1^{-}} \Theta(r \zeta)=\alpha\right\}
$$

and is therefore singular with respect to Lebesgue measure on $\partial \mathbb{D}$. The Clark measure $\mu_{\alpha}$ constructed above can also easily be obtained using the Herglotz Representation Theorem for harmonic functions with positive real part [3, Ch. 9]. As a consequence of this, one can use the fact that $\Theta(0)=0$ to see that $\mu_{\alpha}$ is a probability measure.

It is important to note that the preceding recipe can essentially be reversed. We record this observation here for future reference (see [3, p. 202] for details).

Proposition 2.14. If $\mu$ is a singular probability measure on $\partial \mathbb{D}$, then there is an inner function $\Theta$ with $\Theta(0)=0$ such that the Clark measure for $\Theta$ at $\alpha=1$ is $\mu$. In particular, $\mu$ is the spectral measure for the Clark unitary operator $U_{1}$ on $\mathcal{K}_{\Theta}$.

In the finite-dimensional case, the Clark measures $\mu_{\alpha}$ can be computed explicitly. If $\Theta$ is a finite Blaschke product of order $n$, then $\operatorname{dim} \mathcal{K}_{\Theta}=n$ and the set $E_{\alpha}$ consists of the $n$ distinct points $\zeta_{1}, \zeta_{2}, \ldots, \zeta_{n}$ on $\partial \mathbb{D}$ for which $\Theta\left(\zeta_{j}\right)=\alpha$. The corresponding normalized reproducing kernels $k_{z_{j}}$ satisfy $U_{\alpha} k_{\zeta_{j}}=\zeta_{j} k_{\zeta_{j}}$ for $j=1,2, \ldots, n$ and form an orthonormal basis for the model space $\mathcal{K}_{\Theta}$.

Rank one operators in $\mathcal{T}_{\Theta}$. Let us conclude these preliminaries with a few words concerning truncated Toeplitz operators of rank one. First recall that for each pair $f, g$ of vectors in a Hilbert space $\mathcal{H}$ the operator $f \otimes g: \mathcal{H} \rightarrow \mathcal{H}$ is defined by setting

$$
(f \otimes g)(h):=\langle h, g\rangle f .
$$

Observe that $f \otimes g$ has a rank one and range $\mathbb{C} f$. Moreover, we also have $\|f \otimes g\|=$ $\|f\|\|g\|$. The proof of the next lemma is elementary and is left to the reader.

Lemma 2.16. Let $\mathcal{H}_{1}, \mathcal{H}_{2}$ be Hilbert spaces and let $f_{1}, g_{1} \in \mathcal{H}_{1}$ and $f_{2}, g_{2} \in \mathcal{H}_{2}$ be unit vectors.

(i) If $U: \mathcal{H}_{1} \rightarrow \mathcal{H}_{2}$ is a unitary operator such that

$$
U\left(f_{1} \otimes g_{1}\right) U^{*}=f_{2} \otimes g_{2},
$$

then there exists $a \zeta \in \partial \mathbb{D}$ such that $U f_{1}=\zeta f_{2}$ and $U g_{1}=\zeta g_{2}$. In particular, we have $\left\langle f_{1}, g_{1}\right\rangle_{\mathcal{H}_{1}}=\left\langle f_{2}, g_{2}\right\rangle_{\mathcal{H}_{2}}$. 
(ii) Conversely, if $\left\langle f_{1}, g_{1}\right\rangle=\left\langle f_{2}, g_{2}\right\rangle$, then the operators $f_{1} \otimes g_{1}$ and $f_{2} \otimes g_{2}$ are unitarily equivalent.

The following useful lemma completely characterizes the truncated Toeplitz operators of rank one [23, Thm. 5.1, 7.1]. We remind the reader that $K_{\lambda}$ denotes the reproducing kernel (2.2) for $\mathcal{K}_{\Theta}$ and $C$ denotes the conjugation on $\mathcal{K}_{\Theta}$ from (2.6).

Lemma 2.17 (Sarason). Let $\Theta$ be an inner function.

(i) For each $\lambda \in \mathbb{D}$, the operators $K_{\lambda} \otimes C K_{\lambda}$ and $C K_{\lambda} \otimes K_{\lambda}$ belong to $\mathcal{T}_{\Theta}$.

(ii) If $\eta \in \partial \mathbb{D}$ and $\Theta$ has a $A D C$ at $\eta$, then $K_{\eta} \otimes K_{\eta} \in \mathcal{T}_{\Theta}$.

(iii) The only rank-one operators in $\mathcal{T}_{\Theta}$ are the nonzero scalar multiples of the operators from (i) and (ii).

(iv) If $\Theta$ is a Blaschke product of order $n$, then

(a) $\operatorname{dim} \mathcal{T}_{\Theta}=2 n-1$.

(b) If $\lambda_{1}, \lambda_{2}, \ldots, \lambda_{2 n-1}$ are distinct points of $\mathbb{D}$, then the operators

$$
K_{\lambda_{i}} \otimes C K_{\lambda_{i}}, \quad 1 \leq i \leq 2 n-1,
$$

form a basis for $\mathcal{T}_{\Theta}$.

Elementary complex analysis tells us that the automorphism group Aut $(\mathbb{D})$ of $\mathbb{D}$ can be explicitly presented as

$$
\operatorname{Aut}(\mathbb{D})=\left\{\zeta \varphi_{a}: \zeta \in \partial \mathbb{D}, a \in \mathbb{D}\right\}
$$

where $\varphi_{a}$ denotes the Möbius transformation

$$
\varphi_{a}(z):=\frac{z-a}{1-\bar{a} z} .
$$

For an inner function $\Theta$ with $\Theta \notin \operatorname{Aut}(\mathbb{D})$ we have the following lemma:

Lemma 2.19. Suppose $\Theta$ is inner with $\Theta \notin \operatorname{Aut}(\mathbb{D})$.

(i) If $\lambda_{1}, \lambda_{2} \in \mathbb{D}$, then $K_{\lambda_{1}}$ is not a scalar multiple of $C K_{\lambda_{2}}$.

(ii) If also $\lambda_{1} \neq \lambda_{2}$, then $K_{\lambda_{1}}$ is not a scalar multiple of $K_{\lambda_{2}}$.

(iii) If $\lambda \in \mathbb{D}$, then $K_{\lambda} \otimes C K_{\lambda}$ is not self-adjoint.

Proof. Statements (i) and (ii) are easy computations. For (iii), note that (i) shows that $K_{\lambda} \otimes C K_{\lambda}$ and $\left(K_{\lambda} \otimes C K_{\lambda}\right)^{*}=C K_{\lambda} \otimes K_{\lambda}$ have different ranges.

3. When ARE $\mathcal{T}_{\Theta_{1}}$ AND $\mathcal{T}_{\Theta_{2}}$ SPATIALly isomorphic?

In this section we consider the problem of determining when two spaces $\mathcal{T}_{\Theta_{1}}, \mathcal{T}_{\Theta_{2}}$ of truncated Toeplitz operators are spatially isomorphic. Let us recall the following definition.

Definition 3.1. For $j=1,2$, let $\mathcal{H}_{j}$ be a Hilbert space and $\mathcal{S}_{j}$ be a subspace of $\mathcal{B}\left(\mathcal{H}_{j}\right)$. We say that $\mathcal{S}_{1}$ is spatially isomorphic to $\mathcal{S}_{2}$, written $\mathcal{S}_{1} \cong \mathcal{S}_{2}$, if there is a unitary operator $U: \mathcal{H}_{1} \rightarrow \mathcal{H}_{2}$ so that the map

$$
S \mapsto U S U^{*}, \quad S \in \mathcal{S}_{1},
$$

carries $\mathcal{S}_{1}$ onto $\mathcal{S}_{2}$. In this case we often write $U \mathcal{S}_{1} U^{*}=\mathcal{S}_{2}$. 
Let us be more precise about our main problem. Spatial isomorphisms of the spaces $\mathcal{T}_{\Theta}$ give rise to an equivalence relation on the collection of all inner functions and we wish to determine the structure of the corresponding equivalence classes.

If $\Theta$ is an inner function and $\psi$ belongs to $\operatorname{Aut}(\mathbb{D})$, then the functions $\psi \circ \Theta$ and $\Theta \circ \psi$ are also inner and hence

$$
\mathcal{O}(\Theta):=\left\{\psi_{1} \circ \Theta \circ \psi_{2}: \psi_{1}, \psi_{2} \in \operatorname{Aut}(\mathbb{D})\right\}
$$

consists precisely of those inner functions that can be obtained from $\Theta$ by pre- and post-composition with disk automorphisms. It turns out that while $\Theta_{1} \in \mathcal{O}\left(\Theta_{2}\right)$ is a sufficient condition for ensuring that $\mathcal{T}_{\Theta_{1}} \cong \mathcal{T}_{\Theta_{2}}$, it is not necessary. To formulate the correct theorem, we introduce the conjugation $f \mapsto f^{\#}$ on $H^{2}$ by setting

$$
f^{\#}(z):=\overline{f(\bar{z})}
$$

and we note that $\Theta^{\#}$ is inner if and only if $\Theta$ is. Moreover, note that the \# operation naturally extends to a conjugation on all of $L^{2}$. The main theorem of this section is the following:

Theorem 3.3. For inner functions $\Theta_{1}$ and $\Theta_{2}$,

$$
\mathcal{T}_{\Theta_{1}} \cong \mathcal{T}_{\Theta_{2}} \quad \Leftrightarrow \quad \Theta_{1} \in \mathcal{O}\left(\Theta_{2}\right) \cup \mathcal{O}\left(\Theta_{2}^{\#}\right) .
$$

The proof of the preceding theorem is somewhat long and it requires a number of technical lemmas. We therefore defer the proof until Section 4

It is natural to ask if there are simple geometric conditions on the zeros of Blashke products $B_{1}$ and $B_{2}$ that will ensure that $\mathcal{T}_{B_{1}} \cong \mathcal{T}_{B_{2}}$. While the general question appears difficult, several partial results are available. For instance, if $B_{1}$ and $B_{2}$ are Blaschke products of order 2 , then $\mathcal{T}_{B_{1}} \cong \mathcal{T}_{B_{2}}$ (see Theorem 5.2 below). Another special case is handled by the following corollary. Before presenting it, we require a few words concerning the hyperbolic metric on $\mathbb{D}$.

The hyperbolic (or Poincaré) metric on $\mathbb{D}$ is defined for $z_{1}, z_{2} \in \mathbb{D}$ by

$$
\rho\left(z_{1}, z_{2}\right)=\inf _{\gamma} \int_{\gamma} \frac{2|d z|}{1-|z|^{2}}
$$

where the infimum is taken over all arcs $\gamma$ in $\mathbb{D}$ connecting $z_{1}$ and $z_{2}$. It is wellknown that the hyperbolic metric $\rho$ is conformally invariant in the sense that

$$
\rho\left(z_{1}, z_{2}\right)=\rho\left(\psi\left(z_{1}\right), \psi\left(z_{2}\right)\right), \quad \forall \psi \in \operatorname{Aut}(\mathbb{D}) .
$$

Moreover,

$$
\rho(0, z)=\log \frac{1+|z|}{1-|z|}
$$

and the geodesic through $0, z$ turns out to be $[0, z]$, the line segment from 0 to $z$. The reader can consult [13, p. 4] for further details.

Corollary 3.7. For a finite Blaschke product $B$ of order $n$, we have $\mathcal{T}_{z^{n}} \cong \mathcal{T}_{B}$ if and only if either $B$ has one zero of order $n$ or $B$ has $n$ distinct zeros all lying on a circle $\Gamma$ in $\mathbb{D}$ with the property that if these zeros are ordered according to increasing argument on $\Gamma$, then adjacent zeros are equidistant in the hyperbolic metric (3.5).

Proof. Suppose that $\mathcal{T}_{z^{n}} \cong \mathcal{T}_{B}$. Noting that $\left(z^{n}\right)^{\#}=z^{n}$ and applying Theorem 3.3 we conclude that $B=\psi \circ \varphi^{n}$ for some $\varphi, \psi \in \operatorname{Aut}(\mathbb{D})$. If $\psi$ is a rotation then $B$ 
has one zero of order $n$. If $\psi$ is not a rotation, then the zeros $z_{1}, z_{2}, \ldots, z_{n}$ of $B$ are distinct and satisfy the equation

$$
\left(\frac{z_{j}-a}{1-\bar{a} z_{j}}\right)^{n}=b
$$

for some $a, b \in \mathbb{D}$. The $n$th roots of $b$ are equally spaced on a circle of radius $|b|$ centered at the origin (which are also equally spaced with respect to the hyperbolic metric). The $z_{j}$ are formed by applying a disk automorphism to these $n$th roots of $b$ and thus, by the conformal invariance of the hyperbolic metric, are equally spaced (in the hyperbolic metric) points on some circle $\Gamma$ in $\mathbb{D}$.

Now assume that the zeros $z_{1}, z_{2}, \ldots, z_{n}$ of $B$ satisfy the hypothesis above. If $z_{1}=z_{2}=\cdots=z_{n}$, then $B$ is the $n$th power of a disk automorphism and hence belongs to $\mathcal{O}\left(z^{n}\right)$. In this case, we conclude that $\mathcal{T}_{B} \cong \mathcal{T}_{z^{n}}$. In the second case, map the hyperbolic center of the circle $\Gamma$ to the origin with a disk automorphism $\psi$. The map $\psi$ will also map the circle $\Gamma$ to a circle $|z|=r$ having the same hyperbolic radius as $\Gamma$. Consequently, $\psi$ will map the zeros of $B$ to points $t_{1}, t_{2}, \ldots, t_{n}$ on $|z|=r$ which are equally spaced in the hyperbolic metric. By basic properties of the hyperbolic metric, these points take the form $t_{j}=\omega^{j} a$ where $\omega$ is a primitive $n$th root of unity and $a \in \mathbb{D}$. Putting this all together, we get that the zeros $z_{1}, z_{2}, \cdots, z_{n}$ of $B$ satisfy

$$
z_{j}=\psi^{-1}\left(w^{j} a\right)
$$

and hence

$$
B=\frac{\psi^{n}-a}{1-\bar{a} \psi^{n}} \in \mathcal{O}\left(z^{n}\right) .
$$

By Theorem 3.3 we conclude that $\mathcal{T}_{B} \cong \mathcal{T}_{z^{n}}$.

Remark 3.8. For any inner function $\Theta$, a well-known theorem of Frostman [13, p. 79] implies there are many $\psi \in \operatorname{Aut}(\mathbb{D})$ for which $B=\psi \circ \Theta$ is a Blaschke product. An application of Theorem 3.3 shows that $\mathcal{T}_{\Theta} \cong \mathcal{T}_{B}$. It is natural to ask whether or not there are infinite Blaschke products $B$ for which $\mathcal{T}_{\Theta} \cong \mathcal{T}_{B}$ implies that $\Theta$ is a Blaschke product. Again, using Theorem 3.3. this can be rephrased as: for a fixed infinite Blaschke product $B$, when does $\mathcal{O}(B) \cup \mathcal{O}\left(B^{\#}\right)$ contain only Blaschke products? A little exercise will show that this is true precisely when $\psi \circ B$ is a Blaschke product for every $\psi \in \operatorname{Aut}(\mathbb{D})$. Blaschke products satisfying this property are called indestructible (see [20] and the references therein). It is well-known that Frostman Blaschke products i.e., those Blaschke products $B$ which satisfy

$$
\sup _{\zeta \in \partial \mathbb{D}} \sum_{n=1}^{\infty} \frac{1-\left|a_{n}\right|^{2}}{\left|\zeta-a_{n}\right|}<\infty
$$

where $\left(a_{n}\right)_{n \geq 1}$ are the zeros of $B$, repeated accordingly to multiplicity, are indestructible. Moreover, using a deep theorem of Hruscev and Vinogradov concerning the inner multipliers of the space of Cauchy transforms of measures on the unit circle [3, Ch. 6] along with a result from [17, one can show that $\mathcal{O}(B) \cup \mathcal{O}\left(B^{\#}\right)$ contains only Frostman Blaschke products if and only if $B$ is a Frostman Blaschke product. 


\section{Proof of Theorem 3.3}

The proof of Theorem 3.3 is somewhat lengthy and it is consequently broken up into a series of propositions and lemmas. For the sake of clarity, we deal with the implications $(\Leftarrow)$ and $(\Rightarrow)$ in equation (3.4) separately.

Proof of the implication $(\Leftarrow)$ in $(3.4)$. This is the simpler portion of the proof and it boils down to several computational results.

Proposition 4.1. If $\Theta$ is inner and $\psi \in \operatorname{Aut}(\mathbb{D})$, then $\mathcal{T}_{\Theta} \cong \mathcal{T}_{\Theta \circ \psi}$.

Proof. Let

$$
\psi(z)=\eta \frac{z-a}{1-\bar{a} z}, \quad \eta \in \partial \mathbb{D}, a \in \mathbb{D},
$$

be a typical disk automorphism and define $U: H^{2} \rightarrow H^{2}$ by

$$
U f=\sqrt{\psi^{\prime}}(f \circ \psi)
$$

One can check by the change of variables formula that $U$ is a unitary operator and

$$
U^{-1} f=U^{*} f=\sqrt{\left(\psi^{-1}\right)^{\prime}}\left(f \circ \psi^{-1}\right) .
$$

Next observe that if $f \in \mathcal{K}_{\Theta}$, then

$$
\langle U f,(\Theta \circ \psi) h\rangle=\left\langle f, U^{*}((\Theta \circ \psi) h)\right\rangle=\left\langle f, \Theta \sqrt{\left(\psi^{-1}\right)^{\prime}}\left(h \circ \psi^{-1}\right)\right\rangle=0
$$

for all $h \in H^{2}$. Similarly, for $g \in \mathcal{K}_{\Theta \circ \psi}$ we have

$$
\left\langle U^{*} g, \Theta h\right\rangle=\langle g, U(\Theta h)\rangle=\left\langle g, \sqrt{\psi^{\prime}}(\Theta \circ \psi)(h \circ \psi)\right\rangle=0 .
$$

Thus $U \mathcal{K}_{\Theta}=\mathcal{K}_{\Theta \circ \psi}$ and hence $U$ restricts to a unitary map from $\mathcal{K}_{\Theta}$ onto $\mathcal{K}_{\Theta \circ \psi}$, which we also denote by $U$.

If $A_{g}^{\Theta} \in \mathcal{T}_{\Theta}$, then observe that for $f \in \mathcal{K}_{\Theta}$ we have

$$
\begin{array}{rlr}
{\left[U A_{g}^{\Theta} f\right](\lambda)} & =\left[U P_{\Theta}(g f)\right](\lambda) & \\
& =\sqrt{\psi^{\prime}(\lambda)}\left[P_{\Theta}(g f)\right](\psi(\lambda)) & \text { by (2.4) } \\
& =\sqrt{\psi^{\prime}(\lambda)}\left\langle g f, k_{\psi(\lambda)}\right\rangle \\
& =\sqrt{\psi^{\prime}(\lambda)} \int_{\partial \mathbb{D}} g(\zeta) f(\zeta)\left(\frac{1-\Theta(\psi(\lambda)) \overline{\Theta(\zeta)}}{1-\bar{\zeta} \psi(\lambda)}\right) \frac{|d \zeta|}{2 \pi}
\end{array}
$$

Now make the change of variables $\zeta=\psi(w)$ and use the identities

$$
\psi(z)=\eta \frac{z-a}{1-\bar{a} z}, \quad \psi^{\prime}(z)=\eta \frac{1-|a|^{2}}{(1-\bar{a} z)^{2}}
$$

to show that the above is equal to

$$
\int_{\partial \mathbb{D}} \sqrt{\psi^{\prime}(w)} g(\psi(w)) f(\psi(w)) \frac{1-\Theta(\psi(\lambda)) \overline{\Theta(\psi(w))}}{1-\bar{w} \lambda} \frac{|d w|}{2 \pi}=\left[A_{g \circ \psi}^{\Theta \circ \psi} U f\right](\lambda) .
$$

From this we conclude that $U A_{g}^{\Theta}=A_{g \circ \psi}^{\Theta \circ \psi} U$ whence $A \mapsto U A U^{*}$ is a spatial isomorphism between $\mathcal{T}_{\Theta}$ and $\mathcal{T}_{\Theta \circ \psi}$.

The computational portion of the following proposition is originally due to Crofoot [7. A detailed discussion of these so-called Crofoot transforms in the context of truncated Toeplitz operators can be found in [23, Sec. 13]. 
Proposition 4.3 (Crofoot). If $\Theta$ is inner, $a \in \mathbb{D}$, and $\varphi_{a}$ denotes the Möbius transformation (2.18), then

$$
U f:=\frac{\sqrt{1-|a|^{2}}}{1-\bar{a} \Theta} f
$$

defines a unitary operator from $\mathcal{K}_{\Theta}$ to $\mathcal{K}_{\varphi_{a} \circ \Theta}$. Moreover, $U \mathcal{T}_{\Theta} U^{*}=\mathcal{T}_{\varphi_{a} \circ \Theta}$. Thus for any $\psi \in \operatorname{Aut}(\mathbb{D})$ we have $\mathcal{T}_{\Theta} \cong \mathcal{T}_{\psi \circ \Theta}$.

Our next goal is to establish that $\mathcal{T}_{\Theta} \cong \mathcal{T}_{\Theta}$. This is the content of Proposition 4.6 below. We should remark that this observation is closely related to [1, Cor. 1.7, Prop. 1.8]. The proof of Proposition 4.6 requires two preliminary lemmas. First, recall the definitions (2.6) of the conjugation $C$ on the model space $\mathcal{K}_{\Theta}$ and (3.2) of the conjugation $f \mapsto f^{\#}$. Now let $C^{\#}$ denote the corresponding conjugation on the model space $\mathcal{K}_{\Theta \#}$. Finally, we define a conjugate-linear map $J$ on $\mathcal{K}_{\Theta}$ by $J f=f^{\#}$.

Lemma 4.4. For $\Theta$ inner,

(i) $J \mathcal{K}_{\Theta}=\mathcal{K}_{\Theta \#}$.

(ii) If $g \in \mathcal{K}_{\Theta \#}$, then $J^{-1} g=g^{\#}$.

(iii) $J C: \mathcal{K}_{\Theta} \rightarrow \mathcal{K}_{\Theta \#}$ is unitary. Also, the following formulae hold

$$
J C=C^{\#} J, \quad(J C)^{*}=C J^{-1}=J^{-1} C^{\#} .
$$

(iv) For all $\lambda \in \mathbb{D}$, we have

$$
J C K_{\lambda}^{\Theta}=C^{\#} K_{\bar{\lambda}}^{\Theta^{\#}}, \quad J C\left(C K_{\lambda}^{\Theta}\right)=K_{\bar{\lambda}}^{\Theta^{\#}} .
$$

Proof. Statement (i) follows from the fact that $f \mapsto f^{\#}$ is a conjugation on $H^{2}$ and hence

$$
0=\langle f, \Theta h\rangle=\left\langle\Theta^{\#} h^{\#}, f^{\#}\right\rangle, \quad f \in \mathcal{K}_{\Theta}, h \in H^{2} .
$$

Statement (ii) is immediate since $f \rightarrow f^{\#}$ is an involution on $H^{2}$. For (iii), it is clear that $J C$ is unitary since $J$ and $C$ are isometric and conjugate linear. The remaining identities in (iii) can be easily checked. For (iv) first compute $J K_{\lambda}^{\Theta}=K_{\frac{\Theta}{\lambda}}^{\#}$ and finish by using $J C=C^{\#} J$.

Lemma 4.5. If $A_{\varphi}^{\Theta} \in \mathcal{T}_{\Theta}$, then $J A_{\varphi}^{\Theta} J^{-1}=A_{\varphi}^{\Theta \#}$.

Proof. For all $f, g \in \mathcal{K}_{\Theta \#}^{\infty}$,

$$
\begin{aligned}
\left\langle J A_{\varphi}^{\Theta} J^{-1} f, g\right\rangle & =\left\langle J g, A_{\varphi}^{\Theta} J^{-1} f\right\rangle \\
& =\left\langle\left(A_{\varphi}^{\Theta}\right)^{*} J g, J^{-1} f\right\rangle \\
& =\int_{0}^{2 \pi} \overline{\varphi\left(e^{i \theta}\right) g\left(e^{-i \theta}\right)} f\left(e^{-i \theta}\right) \frac{d \theta}{2 \pi} \\
& =\int_{0}^{-2 \pi} \overline{\varphi\left(e^{-i \theta}\right) g\left(e^{i \theta}\right)} f\left(e^{i \theta}\right) \frac{-d \theta}{2 \pi} \\
& =\int_{0}^{2 \pi} \overline{\varphi\left(e^{-i \theta}\right) g\left(e^{i \theta}\right)} f\left(e^{i \theta}\right) \frac{d \theta}{2 \pi} \\
& =\left\langle\varphi^{\#} f, g\right\rangle \\
& =\left\langle A_{\varphi^{\#}}^{\Theta} f, g\right\rangle .
\end{aligned}
$$


Armed now with Lemmas 4.4 and 4.5 we are ready to prove the following.

Proposition 4.6. For $\Theta$ inner, $\mathcal{T}_{\Theta} \cong \mathcal{T}_{\Theta}$.

Proof. From Lemma 4.4, the operator

$$
J C: \mathcal{K}_{\Theta} \rightarrow \mathcal{K}_{\Theta}{ }^{\#},
$$

is unitary. Furthermore, for $f, g \in \mathcal{K}_{\Theta \#}$ we have

$$
\begin{aligned}
\left\langle(J C) A_{\varphi}^{\Theta}(J C)^{*} f, g\right\rangle & =\left\langle C^{\#} J A_{\varphi}^{\Theta} J^{-1} C^{\#} f, g\right\rangle & & \text { (by Lemma 4.4) } \\
& =\left\langle C^{\#} A_{\varphi^{\#}}^{\Theta^{\#}} C^{\#} f, g\right\rangle & & \text { (by Lemma 4.5) } \\
& =\left\langle\left(A_{\varphi^{\#}}^{\Theta}\right)^{*} f, g\right\rangle & & \text { (Proposition 2.11) } \\
& =\left\langle A \frac{\Theta^{\#}}{\varphi^{\#}} f, g\right\rangle . & &
\end{aligned}
$$

It follows that $A \mapsto(J C) A(J C)^{*}$ is a spatial isomorphism from $\mathcal{T}_{\Theta}$ onto $\mathcal{T}_{\Theta}$.

Propositions 4.1, 4.3, and 4.6 yield the implication $(\Leftarrow)$ of 3.4 . This completes the first part of the proof of Theorem 3.3 .

Technical Lemmas. The proof of the $(\Rightarrow)$ implication in (3.4) is significantly more involved than the proof of $(\Leftarrow)$. We require several additional technical lemmas which we present in this subsection.

Lemma 4.8. Let $\Theta$ be inner, $\Theta \notin \operatorname{Aut}(\mathbb{D})$, and let

$$
\begin{aligned}
& \mathcal{L}_{\Theta}:=\left\{\rho k_{\lambda}: \rho \in \partial \mathbb{D}, \lambda \in \mathbb{D}\right\}, \\
& \widetilde{\mathcal{L}}_{\Theta}:=\left\{\rho C k_{\lambda}: \rho \in \partial \mathbb{D}, \lambda \in \mathbb{D}\right\} .
\end{aligned}
$$

For each fixed $\lambda_{0} \in \mathbb{D}$, we have

$$
\begin{aligned}
\operatorname{dist}\left(k_{\lambda_{0}}, \widetilde{\mathcal{L}}_{\Theta}\right) & >0, \\
\operatorname{dist}\left(C k_{\lambda_{0}}, \mathcal{L}_{\Theta}\right) & >0 .
\end{aligned}
$$

Proof. Suppose that dist $\left(k_{\lambda_{0}}, \widetilde{\mathcal{L}}_{\Theta}\right)=0$ holds for some $\lambda_{0} \in \mathbb{D}$. It follows that there are sequences $\left(\mu_{n}\right)_{n \geq 1} \subset \mathbb{D}$ and $\left(\rho_{n}\right)_{n \geq 1} \subset \partial \mathbb{D}$ so that

$$
\rho_{n} C k_{\mu_{n}} \rightarrow k_{\lambda_{0}}
$$

in the norm of $H^{2}$. Passing to a subsequence, we can assume that $\mu_{n}$ converges to some $\mu_{0} \in \mathbb{D}^{-}$. There are two cases we must consider.

Case 1: If $\mu_{0} \in \mathbb{D}$, then

$$
C k_{\mu_{n}} \rightarrow C k_{\mu_{0}}
$$

in $H^{2}$ and hence pointwise in $\mathbb{D}$. This forces the sequence $\rho_{n}$ to converge to some $\rho_{0} \in \partial \mathbb{D}$ and hence

$$
k_{\lambda_{0}}=\rho_{0} C k_{\mu_{0}} .
$$

However, this contradicts Lemma 2.19 from which we conclude that $\mu_{0} \in \partial \mathbb{D}$.

Case 2: If $\mu_{0} \in \partial \mathbb{D}$, then the sequence $\Theta\left(\mu_{n}\right)$ is bounded and hence upon passing to a subsequence we may assume that $\Theta\left(\mu_{n}\right) \rightarrow a$ for some $a \in \mathbb{D}^{-}$. By (4.11) it follows that

$$
\rho_{n} \frac{\Theta(z)-\Theta\left(\mu_{n}\right)}{\left(z-\mu_{n}\right)\left\|C K_{\mu_{n}}\right\|} \stackrel{H^{2}}{\rightarrow} \frac{1-\overline{\Theta\left(\lambda_{0}\right)} \Theta(z)}{\left(1-\overline{\lambda_{0}} z\right)\left\|K_{\lambda_{0}}\right\|}
$$


whence we also have pointwise convergence on $\mathbb{D}$. For any fixed $z_{0} \in \mathbb{D}$ for which $\Theta\left(z_{0}\right) \neq a$ we conclude that

$$
\rho_{n} \frac{\Theta\left(z_{0}\right)-\Theta\left(\mu_{n}\right)}{\left(z_{0}-\mu_{n}\right)\left\|C K_{\mu_{n}}\right\|} \rightarrow \frac{1-\overline{\Theta\left(\lambda_{0}\right)} \Theta\left(z_{0}\right)}{\left(1-\overline{\lambda_{0}} z_{0}\right)\left\|K_{\lambda_{0}}\right\|} \neq 0 .
$$

But since

$$
\frac{\Theta\left(z_{0}\right)-\Theta\left(\mu_{n}\right)}{z_{0}-\mu_{n}} \rightarrow \frac{\Theta\left(z_{0}\right)-a}{z_{0}-\mu_{0}} \neq 0,
$$

it follows that $\rho_{n}$ converges to some $\rho_{0} \in \partial \mathbb{D}$ and $\left\|C K_{\mu_{n}}\right\|^{-1}$ converges to some finite number $M$. Upon letting $n \rightarrow \infty$ in (4.12), we obtain

$$
\rho_{0} M \frac{\Theta(z)-a}{z-\mu_{0}}=\frac{1-\overline{\Theta\left(\lambda_{0}\right)} \Theta(z)}{\left(1-\overline{\lambda_{0}} z\right)\left\|K_{\lambda_{0}}\right\|} .
$$

Solving for $\Theta(z)$ in the preceding reveals that $\Theta$ is a linear fractional transformation - contradicting the assumption that $\Theta \notin \operatorname{Aut}(\mathbb{D})$. This establishes (4.9). The second inequality (4.10) follows immediately since $C$ is an involutive isometry and so

$$
\operatorname{dist}\left(k_{\lambda_{0}}, \widetilde{\mathcal{L}}_{\Theta}\right)=\operatorname{dist}\left(C k_{\lambda_{0}}, C^{2} \mathcal{L}_{\Theta}\right)=\operatorname{dist}\left(C k_{\lambda_{0}}, \mathcal{L}_{\Theta}\right) .
$$

We henceforth assume that $\Theta_{1}$ and $\Theta_{2}$ are fixed inner functions, neither in $\operatorname{Aut}(\mathbb{D})$, and that $U \mathcal{T}_{\Theta_{1}} U^{*}=\mathcal{T}_{\Theta_{2}}$ for some unitary $U: \mathcal{K}_{\Theta_{1}} \rightarrow \mathcal{K}_{\Theta_{2}}$. We let $C_{1}, C_{2}$ denote the conjugations (2.6) on $\mathcal{K}_{\Theta_{1}}$ and $\mathcal{K}_{\Theta_{2}}$, respectively. To simplify our notation somewhat, we set

$$
k_{\lambda}:=k_{\lambda}^{\Theta_{1}}, \quad \widetilde{k}_{\lambda}:=C_{1} k_{\lambda}^{\Theta_{1}}, \quad \ell_{\lambda}:=k_{\lambda}^{\Theta_{2}}, \quad \tilde{\ell}_{\lambda}:=C_{2} k_{\lambda}^{\Theta_{2}}
$$

for $\lambda \in \mathbb{D}$.

We now exploit the fact that the rank-one operators in $\mathcal{T}_{\Theta_{1}}$ are carried onto the rank-one operators in $\mathcal{T}_{\Theta_{2}}$ by our spatial isomorphism. By Lemma 2.17] and Lemma 2.19, we conclude that $U\left(k_{\lambda} \otimes \widetilde{k}_{\lambda}\right) U^{*}$ is either $\zeta \ell_{\eta} \otimes \tilde{\ell}_{\eta}$ for some $\zeta \in \partial \mathbb{D}$ and $\eta \in \mathbb{D}$, or $\zeta^{\prime} \widetilde{\ell}_{\eta^{\prime}} \otimes \ell_{\eta^{\prime}}$ for some $\zeta^{\prime} \in \partial \mathbb{D}$ and $\eta^{\prime} \in \mathbb{D}$. Upon applying Lemma 2.16 we observe that

$$
U k_{\lambda} \in \mathcal{L}_{\Theta_{2}} \cup \widetilde{\mathcal{L}}_{\Theta_{2}}
$$

In fact, even more is true.

Lemma 4.14. Either $U \mathcal{L}_{\Theta_{1}}=\mathcal{L}_{\Theta_{2}}$ or $U \mathcal{L}_{\Theta_{1}}=\widetilde{\mathcal{L}}_{\Theta_{2}}$. As a consequence, there are maps $w: \mathbb{D} \rightarrow \partial \mathbb{D}$ and $\varphi: \mathbb{D} \rightarrow \mathbb{D}$ so that either

$$
U\left(k_{\lambda} \otimes \widetilde{k}_{\lambda}\right)=w(\lambda) \ell_{\varphi(\lambda)} \otimes \widetilde{\ell}_{\varphi(\lambda)}, \quad \forall \lambda \in \mathbb{D},
$$

or

$$
U\left(k_{\lambda} \otimes \widetilde{k}_{\lambda}\right)=w(\lambda) \tilde{\ell}_{\varphi(\lambda)} \otimes \ell_{\varphi(\lambda)}, \quad \forall \lambda \in \mathbb{D} .
$$

Proof. Since the map $\lambda \mapsto k_{\lambda}$ is continuous from $\mathbb{D}$ to $\mathcal{K}_{\Theta_{1}}$, it follows that

$$
F(\lambda):=U k_{\lambda}
$$

is a continuous function from $\mathbb{D}$ to $\mathcal{K}_{\Theta_{2}}$. Suppose that $F\left(\lambda_{0}\right)=\rho_{0} \ell_{\eta_{0}} \in \mathcal{L}_{\Theta_{2}}$ for some $\lambda_{0}, \eta_{0} \in \mathbb{D}, \rho_{0} \in \partial \mathbb{D}$. We now show that there is an open disk $B\left(\lambda_{0}, \delta\right)$ about $\lambda_{0}$ (of radius $\delta>0$ ) so that

$$
\lambda \in B\left(\lambda_{0}, \delta\right) \quad \Rightarrow \quad U k_{\lambda} \in \mathcal{L}_{\Theta_{2}} .
$$


If this were not the case then by (4.13) there exists sequences $\lambda_{n} \rightarrow \lambda_{0}, \eta_{n} \in \mathbb{D}, \rho_{n} \in$ $\partial \mathbb{D}$ so that $F\left(\lambda_{n}\right)=\rho_{n} \widetilde{\ell}_{\eta_{n}}$. By the continuity of $F$ at $\lambda_{0}$, we see that $\rho_{n} \widetilde{\ell}_{\eta_{n}} \rightarrow \rho_{0} \ell_{\eta_{0}}$, which contradicts Lemma 4.8. Since $\mathbb{D}$ is connected, we conclude that $U \mathcal{L}_{\Theta_{1}} \subset \mathcal{L}_{\Theta_{2}}$.

If we now interchange the roles of $\Theta_{1}$ and $\Theta_{2}$, replacing $U$ with $U^{*}$, the argument above shows that $U^{*} \mathcal{L}_{\Theta_{1}} \subset \mathcal{L}_{\Theta_{1}}$. This means that $\mathcal{L}_{\Theta_{2}} \subset U \mathcal{L}_{\Theta_{1}}$ and so $U \mathcal{L}_{\Theta_{1}}=$ $\mathcal{L}_{\Theta_{2}}$. The same argument shows that if $F\left(\lambda_{0}\right) \in \widetilde{\mathcal{L}}_{\Theta_{2}}$, then $U \mathcal{L}_{\Theta_{1}}=\widetilde{\mathcal{L}}_{\Theta_{2}}$.

Remark 4.15. Now observe that it suffices to consider the case where $U \mathcal{L}_{\Theta_{1}}=$ $\mathcal{L}_{\Theta_{2}}$. Indeed, suppose that $U \mathcal{L}_{\Theta_{1}}=\widetilde{\mathcal{L}}_{\Theta_{2}}$. We know from Proposition 4.6 that $\mathcal{T}_{\Theta_{2}} \cong \mathcal{T}_{\Theta_{2}^{\#}}$ and, from Lemma 4.4 part (iv), the unitary $J C$ implementing this spatial isomorphism carries $\widetilde{\mathcal{L}}_{\Theta_{2}}$ onto $\mathcal{L}_{\Theta_{2}^{\#}}$. By replacing $\Theta_{2}$ with $\Theta_{2}^{\#}$ if necessary (which does not change $\mathcal{O}\left(\Theta_{2}\right) \cup \mathcal{O}\left(\Theta_{2}^{\#}\right)$ ), we assume for the remainder of the proof that $U \mathcal{L}_{\Theta_{1}}=\mathcal{L}_{\Theta_{2}}$. Under this assumption it follows that

$$
U\left(k_{\lambda} \otimes \widetilde{k}_{\lambda}\right)=w(\lambda) \ell_{\varphi(\lambda)} \otimes \widetilde{\ell}_{\varphi(\lambda)}, \quad \forall \lambda \in \mathbb{D},
$$

for some functions $w: \mathbb{D} \rightarrow \partial \mathbb{D}$ and $\varphi: \mathbb{D} \rightarrow \mathbb{D}$.

Lemma 4.17. The function $\varphi$ in (4.16) belongs to Aut $(\mathbb{D})$.

Proof. We first prove that $\varphi: \mathbb{D} \rightarrow \mathbb{D}$ is a bijection. Suppose that $\varphi\left(\lambda_{1}\right)=\varphi\left(\lambda_{2}\right)$. It follows from (4.16) that $k_{\lambda_{1}}=c k_{\lambda_{2}}$ for some scalar $c$. By Lemma 2.19, we conclude that $\lambda_{1}=\lambda_{2}$ whence $\varphi$ is injective. Now let $\eta \in \mathbb{D}$. By Lemma 4.14 we know that

$$
U^{*}\left(\ell_{\eta} \otimes \tilde{\ell}_{\eta}\right) U=c k_{\lambda} \otimes \widetilde{k}_{\lambda}
$$

for some $\lambda \in \mathbb{D}$ and some scalar $c$. We cannot have

$$
U^{*}\left(\ell_{\eta} \otimes \widetilde{\ell}_{\eta}\right) U=c \widetilde{k}_{\lambda} \otimes k_{\lambda}
$$

or else (by Lemma (2.16) $) U k_{\lambda}=c \widetilde{\ell}_{\eta}$ which we are assuming is not the case. Another application of Lemma 2.16 reveals that $\varphi(\lambda)=\eta$ whence $\varphi$ is surjective.

To show that $\varphi \in \operatorname{Aut}(\mathbb{D})$, it suffices to prove that $\varphi$ is analytic on $\mathbb{D}$. We may assume that $\Theta_{2}(0) \neq 0$ and $\Theta_{2}\left(w_{0}\right)=0$ for some $w_{0} \in \mathbb{D}$. If this is not the case, choose $a_{1}, a_{2} \in \mathbb{D}\left(a_{1} \neq a_{2}\right)$ so that $\Theta_{2}\left(a_{1}\right)=\Theta_{2}\left(a_{2}\right)=b$, replace $\Theta_{2}$ by $\varphi_{b} \circ \Theta_{2} \circ \varphi_{-a}$, and appeal to Propositions 4.1 and 4.3 . In particular, this means that if $L_{\eta}$ denotes the reproducing kernel for $\mathcal{K}_{\Theta_{2}}$, then

$$
L_{0}=1, \quad L_{w_{0}}=\frac{1}{1-\overline{w_{0}} z} .
$$

Let $f=U^{-1} L_{0}$ and $g=U^{-1} L_{w_{0}}$. Then for any $\lambda \in \mathbb{D}$ we have

$$
\begin{aligned}
f(\lambda) & =\left\langle f, K_{\lambda}\right\rangle \\
& =\left\langle U f, U K_{\lambda}\right\rangle \\
& =\left\langle 1, \frac{w(\lambda)\left\|K_{\lambda}\right\|}{\left\|L_{\varphi(\lambda)}\right\|} L_{\varphi(\lambda)}\right\rangle \\
& =\frac{\frac{w(\lambda)}{\left\|K_{\lambda}\right\|}}{\left\|L_{\varphi(\lambda)}\right\|} .
\end{aligned}
$$

Similarly, using the formula for $f(\lambda)$ above, we get

$$
\begin{aligned}
g(\lambda) & =\left\langle g, K_{\lambda}\right\rangle \\
& =\left\langle U g, U K_{\lambda}\right\rangle
\end{aligned}
$$




$$
\begin{aligned}
& =\left\langle\frac{1}{1-\overline{w_{0}} z}, \frac{w(\lambda)\left\|K_{\lambda}\right\|}{\left\|L_{\varphi(\lambda)}\right\|} L_{\varphi(\lambda)}\right\rangle \\
& =\frac{1}{1-\overline{w_{0}} \varphi(\lambda)} \frac{\frac{w(\lambda)}{\left\|K_{\lambda}\right\|}}{\left\|L_{\varphi(\lambda)}\right\|} \\
& =\frac{1}{1-\overline{w_{0}} \varphi(\lambda)} f(\lambda) .
\end{aligned}
$$

Since the functions $f$ and $g$ are analytic (and not identically zero) on $\mathbb{D}$, upon solving for $\varphi(\lambda)$ in the preceding identity we conclude that $\varphi$ is analytic on $\mathbb{D}$.

Proof of the implication $(\Rightarrow)$ in (3.4). We have already seen via Propositions 4.1, 4.3, and 4.6 that

$$
\Theta_{1} \in \mathcal{O}\left(\Theta_{2}\right) \cup \mathcal{O}\left(\Theta_{2}^{\#}\right) \quad \Rightarrow \quad \mathcal{T}_{\Theta_{1}} \cong \mathcal{T}_{\Theta_{2}} .
$$

We now prove the reverse implication. In light of Remark4.15 and Lemma 4.17 we may assume that

$$
U k_{\lambda}=w(\lambda) \ell_{\varphi(\lambda)}, \quad \forall \lambda \in \mathbb{D},
$$

for some functions $w: \mathbb{D} \rightarrow \partial \mathbb{D}$ and $\varphi \in \operatorname{Aut}(\mathbb{D})$. Consequently we may appeal to Lemma 2.16 to conclude that

$$
U\left(k_{\lambda} \otimes \tilde{k}_{\lambda}\right) U^{*}=w(\lambda) \ell_{\varphi(\lambda)} \otimes \tilde{\ell}_{\varphi(\lambda)} .
$$

Upon taking adjoints in the preceding equation we then obtain

$$
U\left(\widetilde{k}_{\lambda} \otimes k_{\lambda}\right) U^{*}=\overline{w(\lambda)} \tilde{\ell}_{\varphi(\lambda)} \otimes \ell_{\varphi(\lambda)} .
$$

Lemma 2.16 now yields

$$
U \widetilde{k}_{\lambda}=w(\lambda) \tilde{\ell}_{\varphi(\lambda)} .
$$

Next we combine (4.18) and (4.19) to obtain

$$
\left|\left\langle\widetilde{k}_{\lambda}, k_{\lambda}\right\rangle\right|=\left|\left\langle\widetilde{\ell}_{\varphi(\lambda)}, \ell_{\varphi(\lambda)}\right\rangle\right| .
$$

Noting that

$$
k_{\lambda}=\frac{K_{\lambda}}{\left\|K_{\lambda}\right\|}, \quad\left\langle C K_{\lambda}, K_{\lambda}\right\rangle=\Theta^{\prime}(\lambda), \quad\left\|C K_{\lambda}\right\|=\left\|K_{\lambda}\right\|=\sqrt{\frac{1-|\Theta(\lambda)|^{2}}{1-|\lambda|^{2}}}
$$

we get

$$
\frac{\left|\Theta_{1}^{\prime}(\lambda)\right|\left(1-|\lambda|^{2}\right)}{1-\left|\Theta_{1}(\lambda)\right|^{2}}=\frac{\left|\Theta_{2}^{\prime}(\varphi(\lambda))\right|\left(1-|\varphi(\lambda)|^{2}\right)}{1-\left|\Theta_{2}(\varphi(\lambda))\right|^{2}} .
$$

Using the Schwarz-Pick lemma [13, p. 2] we have

$$
\left|\varphi^{\prime}(z)\right|=\frac{1-|\varphi(z)|^{2}}{1-|z|^{2}}, \quad \forall z \in \mathbb{D}, \quad \forall \varphi \in \operatorname{Aut}(\mathbb{D}),
$$

whence the identity (4.20) becomes

$$
\frac{\left|\Theta_{1}^{\prime}(\lambda)\right|}{1-\left|\Theta_{1}(\lambda)\right|^{2}}=\frac{\left|\Theta_{2}^{\prime}(\varphi(\lambda))\right|}{1-\left|\Theta_{2}(\varphi(\lambda))\right|^{2}}\left|\varphi^{\prime}(\lambda)\right| .
$$

Replacing $\Theta_{2}$ by $\Theta_{2} \circ \varphi$ in the preceding formula gives us

$$
\frac{\left|\Theta_{1}^{\prime}(\lambda)\right|}{1-\left|\Theta_{1}(\lambda)\right|^{2}}=\frac{\left|\Theta_{2}^{\prime}(\lambda)\right|}{1-\left|\Theta_{2}(\lambda)\right|^{2}} .
$$


Another application of the Schwarz-Pick lemma shows that (4.21) continues to hold if $\Theta_{1}$ is replaced by $\psi \circ \Theta_{1}$ for all $\psi \in \operatorname{Aut}(\mathbb{D})$. It follows that we may assume that

$$
\Theta_{1}(0)=\Theta_{2}(0)=0, \quad \Theta_{1}^{\prime}(0) \neq 0, \quad \Theta_{2}^{\prime}(0) \neq 0 .
$$

If not, choose $a \in \mathbb{D}$ so that $\Theta_{1}^{\prime}(a) \neq 0$ and $\Theta_{2}^{\prime}(a) \neq 0$. Let $b_{1}=\Theta_{1}\left(\varphi_{-a}(0)\right)$ and $b_{2}=\Theta_{2}\left(\varphi_{-a}(0)\right)$. Now replace $\Theta_{1}$ by $\varphi_{b_{1}} \circ \Theta_{1} \circ \varphi_{-a}$ and $\Theta_{2}$ by $\varphi_{b_{2}} \circ \Theta_{2} \circ \varphi_{-a}$ and observe that (4.22) still holds. It is important to note that all of these simplifying assumptions on $\Theta_{2}$ has not altered $\mathcal{O}\left(\Theta_{2}\right) \cup \mathcal{O}\left(\Theta_{2}^{\#}\right)$.

The assumption (4.22) means that both $\Theta_{1}$ and $\Theta_{2}$ are invertible near the origin. Thus there is an $\varepsilon>0$ such that $\Theta_{1}$ and $\Theta_{2}$ are injective on the disk $B(0, \varepsilon)$. There is also a $\delta>0$ with $B(0, \delta) \subset \Theta_{1}(B(0, \varepsilon))$ and $B(0, \delta) \subset \Theta_{2}(B(0, \varepsilon))$.

Now suppose that $|z|<\delta$. Then $\Theta_{1}^{-1}([0, z])$ is a curve $\gamma$ in $B(0, \varepsilon)$ and $\Theta_{2} \circ$ $\Theta_{1}^{-1}([0, z])=\Theta_{2}(\gamma)$ is a curve $\Gamma$ in $B(0, \delta)$ going from 0 to $\beta:=\Theta_{2} \circ \Theta_{1}^{-1}(z)$. From our discussion in the previous paragraph along with the change of variables formula and (4.21) we get

$$
\int_{\gamma} \frac{\left|\Theta_{1}^{\prime}(t)\right|}{1-\left|\Theta_{1}(t)\right|^{2}} d t=\int_{\gamma} \frac{\left|\Theta_{2}^{\prime}(t)\right|}{1-\left|\Theta_{2}(t)\right|^{2}} d t=\int_{\Gamma} \frac{|d w|}{1-|w|^{2}} .
$$

Thus $\rho(0, z) \geq \rho(0, \beta)$ whence, by (3.6),$|z| \geq|\beta|$ and so

$$
\left|\Theta_{2} \circ \Theta_{1}^{-1}(z)\right| \leq|z|
$$

for small $|z|$. A similar argument also shows that $\left|\Theta_{1} \circ \Theta_{2}^{-1}\right| \leq|z|$ for small $|z|$. Putting this all together we find that

$$
|z|=\left|\Theta_{2} \circ \Theta_{1}^{-1}(z)\right|, \quad \forall|z|<\delta
$$

and hence there is a $\zeta \in \partial \mathbb{D}$ such that

$$
\Theta_{2} \circ \Theta_{1}^{-1}(z)=\zeta z, \quad \forall|z|<\delta .
$$

Replacing $z$ by $\Theta_{1}(z)$ for $|z|$ small, we have $\Theta_{2}(z)=\zeta \Theta_{1}(z)$ and so $\Theta_{2}=\zeta \Theta_{1}$ on $\mathbb{D}$. Thus $\Theta_{1} \in \mathcal{O}\left(\Theta_{2}\right)$ as desired. This completes the proof of Theorem 3.3

\section{Unitary equivalence to a truncated Toeplitz operator}

In this section we attempt to describe those classes of Hilbert space operators which are UETTO (unitarily equivalent to a truncated Toeplitz operator). This question is more subtle that it might at first appear. For instance, the Volterra integration operator, being the Cayley transform of the compressed shift $A_{z}$ on a certain model space, is UETTO [21] (see also [18, p. 41]). While the general question appears quite difficult, we are able to obtain concrete results in a few specific cases.

Theorem 5.1. Every rank one operator is UETTO.

Proof. Let $T=u \otimes v$ be a rank one operator on an $n$-dimensional Hilbert space. Without loss of generality, suppose that $2 \leq n \leq \infty,\|u\|=\|v\|=1$ and

$$
0 \leq\langle u, v\rangle \leq 1 .
$$

We claim that there exists a Blaschke product $\Theta$ of order $n$ (i.e., having $n$ zeros, counting according to multiplicity) and an appropriate $\lambda$ so that $u \otimes v$ is unitarily equivalent to a multiple of $k_{\lambda} \otimes C k_{\lambda}$. By Lemmas 2.16 and 2.17 it suffices to exhibit $\Theta$ and $\lambda$ so that

$$
\langle u, v\rangle=\left\langle k_{\lambda}, C k_{\lambda}\right\rangle .
$$


There are three cases to consider:

(i) Suppose that $\langle u, v\rangle=0$. In this case let $\Theta$ be a Blaschke product of order $n$ having a repeated root at $\lambda=0$. Then

$$
\left\langle k_{0}, C k_{0}\right\rangle=\left\langle 1, \frac{\Theta}{z}\right\rangle=\overline{\Theta^{\prime}(0)}=0=\langle u, v\rangle
$$

as desired.

(ii) Suppose that $\langle u, v\rangle=1$. Since $u$ and $v$ are unit vectors, it follows that $u=v$. In this case, let $\Theta$ be a Blaschke product of order $n$ having an ADC at $\lambda=1$ and satisfying $\Theta(1)=1$ in the non-tangential limiting sense. A short computation shows that $C k_{1}=k_{1}$ whence

$$
\left\langle k_{1}, C k_{1}\right\rangle=1=\langle u, v\rangle
$$

as desired.

(iii) Suppose that $0<\langle u, v\rangle<1$. In this case, let $\Theta$ be a Blaschke product of order $n$ with a simple root at $\lambda=0$ and having its remaining roots $\lambda_{i}$ being strictly positive. In this case

$$
\left\langle k_{0}, C k_{0}\right\rangle=\Theta^{\prime}(0)=\prod_{i=1}^{n} \lambda_{i} .
$$

By selecting the zeros $\lambda_{i}$ appropriately, the preceding can be made to equal $\langle u, v\rangle$ as was required.

Theorem 5.2. Every $2 \times 2$ matrix is UETTO. In fact, if $T$ is a given $2 \times 2$ matrix and $\Theta$ is a Blaschke product of order 2 , then $\mathcal{T}_{\Theta}$ contains an operator unitarily equivalent to $T$.

Proof. Let $T$ be a given $2 \times 2$ matrix and let $\Theta$ be a Blaschke product of order 2 . Using the fact that a $2 \times 2$ matrix is unitarily equivalent to a complex symmetric matrix (see [2, Cor. 3.3], [1, Ex. 6], or [26, Cor. 3]), we may restrict our attention to the case where $T$ is complex symmetric: $T=T^{t}$. Now observe that the subspace of $S_{2}(\mathbb{C}) \subset M_{2}(\mathbb{C})$ consisting of all $2 \times 2$ complex symmetric matrices has dimension 3. Next note that part (iv) of Lemma 2.17 asserts that $\operatorname{dim} \mathcal{T}_{\Theta}=3$ as well. If $\beta$ is a $C$-real orthonormal basis for $\mathcal{K}_{\Theta}$ (see [10, Lem. 2.6] for details), then the map $\Phi: \mathcal{T}_{\Theta} \rightarrow S_{2}(\mathbb{C})$ defined by $\Phi(A)=[A]_{\beta}$ is clearly injective whence its image contains $T$ [10, Lem. 2.7].

Corollary 5.3. If $\Theta_{1}$ and $\Theta_{2}$ are Blaschke products of order 2 , then $\mathcal{T}_{\Theta_{1}} \cong \mathcal{T}_{\Theta_{2}}$.

Proof. The proof of Theorem 5.2 provides a recipe for constructing spatial isomorphisms $\Phi_{1}: \mathcal{T}_{\Theta_{1}} \rightarrow S_{2}(\mathbb{C})$ and $\Phi_{2}: \mathcal{T}_{\Theta_{2}} \rightarrow S_{2}(\mathbb{C})$. It follows that $\Phi_{2} \circ \Phi_{1}: \mathcal{T}_{\Theta_{1}} \rightarrow$ $\mathcal{T}_{\Theta_{2}}$ is a spatial isomorphism.

Theorem 5.4. If $N$ is an $n \times n$ normal matrix and $\Theta$ is a Blaschke product of order $n$, then $N$ is unitarily equivalent to an operator in $\mathcal{T}_{\Theta}$.

Proof. By the Spectral Theorem, we know that $N$ is unitarily equivalent to the diagonal matrix $\operatorname{diag}\left(\lambda_{1}, \lambda_{2}, \ldots, \lambda_{n}\right)$ where $\lambda_{1}, \lambda_{2}, \ldots, \lambda_{n}$ denote the eigenvalues of $N$, repeated according to their multiplicity. Select a Clark unitary operator $U=U_{\alpha}$ (see (2.12) ) and note from Theorem 2.13 that $U \in \mathcal{T}_{\Theta}$ as is $p(U)$ for any polynomial $p(z)$. Also note that the eigenvalues $\zeta_{1}, \zeta_{2}, \ldots, \zeta_{n}$ of $U$ have multiplicity one [5. 
Thm. 3.2] (see also [10, Thm. 8.2]). Thus, there exists a polynomial $p(z)$ such that $p\left(\zeta_{i}\right)=\lambda_{i}$ for $i=1,2, \ldots, n$. It follows that $p(U)$ is unitarily equivalent to $\operatorname{diag}\left(\lambda_{1}, \lambda_{2}, \ldots, \lambda_{n}\right)$ and hence to $N$ itself.

If we are willing to sacrifice the arbitrary selection of $\Theta$, then the preceding can be generalized to the infinite-dimensional setting. To do so, we require some preliminary remarks on multiplication operators. For a compactly supported Borel measure $\mu$ on $\mathbb{C}$, we have the associated algebra

$$
\mathcal{M}_{\mu}:=\left\{M_{\varphi} \in \mathcal{B}\left(L^{2}(\mu)\right): \varphi \in L^{\infty}(\mu)\right\}
$$

of multiplication operators on $L^{2}(\mu)$. For each such measure we define the ordered pair $\kappa(\mu)=(\epsilon, n)$ where

$$
\epsilon= \begin{cases}0 & \text { if } \mu \text { is purely atomic } \\ 1 & \text { otherwise }\end{cases}
$$

and $0 \leq n \leq \infty$ denotes the number of atoms of $\mu$. In terms of the function $\kappa$, the following theorem of Halmos and von Neumann [14] (see also [6, Thm. 7.51.7]) describes when the algebras (5.5) are spatially isomorphic.

Theorem 5.6 (Halmos and von Neumann). For two compactly supported Borel measures $\mu_{1}, \mu_{2}$ on $\mathbb{C}$, the algebras $\mathcal{M}_{\mu_{1}}$ and $\mathcal{M}_{\mu_{2}}$ are spatially isomorphic if and only if $\kappa\left(\mu_{1}\right)=\kappa\left(\mu_{2}\right)$.

Theorem 5.7. Every normal operator on a separable Hilbert space is UETTO.

Proof. If $N$ is a normal operator on a separable Hilbert space, then the spectral theorem asserts that $N$ is unitarily equivalent to $M_{\varphi}: L^{2}(\mu) \rightarrow L^{2}(\mu)$ for some compactly supported Borel measure $\mu$ on $\mathbb{C}$ and some $\varphi \in L^{\infty}(\mu)$. Let $\eta$ be a singular probability measure on $\partial \mathbb{D}$ for which $\kappa(\mu)=\kappa(\eta)$. By Theorem [5.6] $M_{\varphi}: L^{2}(\mu) \rightarrow L^{2}(\mu)$ is unitarily equivalent to $M_{\psi}: L^{2}(\eta) \rightarrow L^{2}(\eta)$, for some $\psi \in L^{\infty}(\eta)$.

By Proposition 2.14, $\eta$ is a Clark measure for some Clark unitary operator $U_{1}$ on $\mathcal{K}_{\Theta}$ for some inner $\Theta$. Again by Proposition 2.14, $U_{1}$ is unitarily equivalent to $\left(M_{z}, L^{2}(\eta)\right)$. Moreover, by Theorem 2.13, we also get that $U_{1}$ as well as $\psi\left(U_{1}\right)$ belong to $\mathcal{T}_{\Theta}$. Finally, note that

$$
\psi\left(U_{1}\right) \cong\left(M_{\psi}, L^{2}(\eta)\right) \cong\left(M_{\varphi}, L^{2}(\mu)\right) \cong N
$$

In the previous line we use $\cong$ to denote unitary equivalence of two operators.

Theorem 5.8. For $k \in \mathbb{N} \cup\{\infty\}$, the $k$-fold inflation of a finite Toeplitz matrix is UETTO.

Proof. Suppose that $n \in \mathbb{N}$ and $A_{\psi} \in \mathcal{T}_{z^{n}}$, where

$$
\psi(\zeta)=\sum_{m=-n+1}^{n-1} a_{m} \zeta^{m}
$$

is a trigonometric polynomial. In particular, the matrix of $A_{\psi}$ relative to the usual monomial basis $\left\{1, z, \ldots, z^{n-1}\right\}$ for $\mathcal{K}_{z^{n}}$ is a Toeplitz matrix and every finite Toeplitz matrix arises in this manner.

For $k \in \mathbb{N} \cup\{\infty\}$ let $A_{\psi} \otimes I$ denote the $k$-fold inflation of $A_{\psi}$, where $I$ is the identity matrix on some $k$-dimensional Hilbert space. We will now show that $A_{\psi} \otimes I$ 
is UETTO. To do this let $B$ be a Blaschke product of order $k$ (Note that $k$ can be infinite). If $T_{B}$ denotes the usual Toeplitz operator on $H^{2}$ with symbol $B$, then

$$
T_{B}\left(B^{j} \mathcal{K}_{B}\right)=B^{j+1} \mathcal{K}_{B}, \quad j=0,1,2, \ldots
$$

Since

$$
H^{2}=\bigoplus_{j=0}^{\infty} B^{j} \mathcal{K}_{B},
$$

we see that $T_{B}$ is unitarily equivalent to a shift of multiplicity $k$, i.e., $T_{B} \cong T_{z} \otimes I$ (This is a standard fact from operator theory [6, p. 111]). In a similar way, one shows that

$$
T_{B^{m}} \cong T_{z^{m}} \otimes I, \quad m \in \mathbb{Z},
$$

and so, from (5.9),

$$
T_{\psi(B)} \cong T_{\psi} \otimes I .
$$

A short exercise using the fact that $\mathcal{K}_{B}=\left(B H^{2}\right)^{\perp}$ will show that

$$
\mathcal{K}_{B^{n}}=\bigoplus_{j=0}^{n-1} B^{j} \mathcal{K}_{B}
$$

Combine this with the above discussion to show that $A_{\psi(B)}: \mathcal{K}_{B^{n}} \rightarrow \mathcal{K}_{B^{n}}$ (which is the compression of $T_{\psi(B)}$ to $\left.\mathcal{K}_{B^{n}}\right)$ is unitarily equivalent to $A_{\psi} \otimes I$.

We conclude this section with several open questions. The first two are motivated by Theorem 5.8

Question 5.10. For which truncated Toeplitz operators $A_{\varphi}^{\Theta}$ and for which $k \in$ $\mathbb{N} \cup\{\infty\}$ is the $k$-fold inflation of $A_{\varphi}^{\Theta}$ UETTO?

Question 5.11. When is the direct sum of truncated Toeplitz operators UETTO?

It is known that every truncated Toeplitz operator is a complex symmetric operator (see Definition 2.10 and Proposition 2.11). Moreover, so is the Volterra integration operator, every $2 \times 2$ matrix, and every normal operator [10, 11]. In light of the results obtained in this section, it is natural to ask the following:

Question 5.12. Which complex symmetric operators are UETTO?

\section{Similarity to a truncated Toeplitz operator}

It was asked in [16] whether or not the inverse Jordan problem can be solved in the class of Toeplitz matrices. That is to say, given any Jordan canonical form, can one find a Toeplitz matrix that is similar to this form? A negative answer to this question was subsequently provided by G. Heinig [15. On the other hand, it turns out that the inverse Jordan structure problem is always solvable in the class of truncated Toeplitz operators. In fact, we get a bit more.

Theorem 6.1. Every operator on a finite dimensional space is similar to a coanalytic truncated Toeplitz operator.

Proof. Recalling the notation (2.18), for a finite Blaschke product $\Theta$, we write

$$
\Theta=\varphi_{z_{1}}^{d_{1}} \varphi_{z_{2}}^{d_{2}} \cdots \varphi_{z_{r}}^{d_{r}},
$$


where $z_{1}, z_{2}, \ldots, z_{r}$ are the distinct zeros of $\Theta$, and $d:=d_{1}+d_{2}+\cdots+d_{r}$ is the order of $\Theta$. Let

$$
\mathcal{Q}:=\left\{A_{\bar{\psi}} \in \mathcal{T}_{\Theta}: \psi \in H^{\infty}\right\}
$$

denote the algebra of co-analytic truncated Toeplitz operators on $\mathcal{K}_{\Theta}$. Note that $\mathcal{Q}$ is the set of $A_{\bar{p}}$ where $p$ is a polynomial of degree at most $d$.

For $1 \leq i \leq r$, let $P_{i}$ be the Riesz idempotent corresponding to the eigenvalue $\bar{z}_{i}$ of $A_{\bar{z}}$ and note that $P_{i} \in \mathcal{Q}$ and $\operatorname{ran} P_{i}=\operatorname{ker}\left(A_{\bar{z}}-\overline{z_{i}} I\right)^{d_{i}}$ [8, p. 569]. From here it is easy to see that

$$
\operatorname{ran} P_{i}=\mathcal{K}_{\varphi_{z_{i}}^{d_{i}}}
$$

and that an orthonormal basis for this subspace is

$$
\left\{k_{z_{i}} \varphi_{z_{i}}^{j-1}: 1 \leq j \leq d_{i}\right\} .
$$

Relative to the basis above, the restriction of $A_{\bar{\varphi}_{z_{i}}}$ to $\mathcal{K}_{\varphi_{z_{i}}^{d_{i}}}$ has a matrix which is a $d_{i} \times d_{i}$ Jordan block. Thus the algebra

$$
\mathcal{Q}_{i}:=\mathcal{Q} \mid \mathcal{K}_{\varphi_{z_{i}}^{d_{i}}}
$$

is spatially isomorphic to the algebra of $d_{i} \times d_{i}$ upper triangular Toeplitz matrices.

Since

$$
\mathcal{K}_{\Theta}=\mathcal{K}_{\varphi_{z_{1}}^{d_{1}}} \oplus \mathcal{K}_{\varphi_{z_{2}}^{d_{2}}} \oplus \cdots \oplus \mathcal{K}_{\varphi_{z_{r}}^{d_{r}}}
$$

is a (non-orthogonal) direct sum of vector spaces, we see from (6.3) that

$$
\mathcal{Q}=\mathcal{Q}_{1} \oplus \mathcal{Q}_{2} \oplus \cdots \oplus \mathcal{Q}_{r}
$$

is a (non-orthogonal) direct sum of algebras. It is now clear that given a Jordan canonical form, we can find a co-analytic truncated Toeplitz operator with that form. The number of blocks in the form is the number of distinct zeros of $\Theta$ and the size of each block determines the multiplicity of each given zero.

The proof of Theorem 6.1 also proves the following corollary:

Corollary 6.4. If $\Theta$ is a finite Blaschke product, $\mathcal{Q}$, the co-analytic truncated operators on $\mathcal{K}_{\Theta}$, is spatially similar to $\mathcal{Q}^{*}:=\left\{A^{*}: A \in \mathcal{Q}\right\}$, the analytic truncated Toeplitz operators on $\mathcal{K}_{\Theta}$.

Proof. Observe that for each $k, \mathcal{Q}_{k}$ and $\left(\mathcal{Q}_{k}\right)^{*}$ are spatially isomorphic.

Theorem 5.7 asserts that for a fixed inner function $\Theta, \mathcal{T}_{\Theta}$ contains many normal operators. However, they are not among the analytic (or co-analytic) truncated Toeplitz operators except in trivial cases.

Proposition 6.5. If $\Theta$ is inner and $A_{\varphi} \in \mathcal{T}_{\Theta}$ is normal and not a multiple of the identity operator, then $\varphi \notin H^{2} \cup \overline{H^{2}}$.

Proof. Suppose that $\varphi \in H^{2}$ and $A_{\varphi} \in \mathcal{T}_{\Theta}$ is normal. Since $A_{\varphi}=A_{P_{\Theta} \varphi}$ [23, Thm. 3.1], we can assume that $\varphi \in \mathcal{K}_{\Theta}$. Furthermore, if $K_{0}=1-\overline{\Theta(0)} \Theta$ is the reproducing kernel for $\mathcal{K}_{\Theta}$ at the origin, we have

$$
A_{K_{0}} f=P_{\Theta}(f-f \overline{\Theta(0)} \Theta)=f, \quad f \in \mathcal{K}_{\Theta},
$$

and so $A_{K_{0}}=I$ (this identity was observed in [23, p. 499]). Since $A_{\varphi}$ is normal if and only if $A_{\varphi}-a I=A_{\varphi-a K_{0}}$ is normal, we can set $a=\varphi(0) /\left\|K_{0}\right\|^{2}$ to assume that $A_{\varphi}$ is normal with

$$
\varphi \in \mathcal{K}_{\Theta} \quad \text { and } \quad \varphi(0)=0 .
$$


This means that $\varphi=z g$ for some $g \in H^{2}$, and, since $S^{*} \varphi=(\varphi-\varphi(0)) / z \in \mathcal{K}_{\Theta}$, we see that $g \in \mathcal{K}_{\Theta}$.

To show that $A_{\varphi}$ cannot be normal, we will prove the inequality

$$
\left\|A_{\varphi}^{*} K_{0}\right\|<\left\|A_{\varphi} K_{0}\right\| \text {. }
$$

Observe that

$$
A_{\varphi} K_{0}=P_{\Theta}(\varphi-\overline{\Theta(0)} \Theta \varphi)=\varphi
$$

since $\varphi \in \mathcal{K}_{\Theta}$. Now notice that

$$
\begin{aligned}
A_{\varphi}^{*} K_{0} & =P_{\Theta}(\bar{\varphi}-\overline{\Theta(0)} \bar{\varphi} \Theta) \\
& =0-\overline{\Theta(0)} P_{\Theta}(\overline{(z g)} \Theta) \\
& =-\overline{\Theta(0)} P_{\Theta}(C g) \\
& =-\overline{\Theta(0)} C g .
\end{aligned}
$$

Finally note that

$$
\begin{aligned}
\left\|A_{\varphi}^{*} K_{0}\right\| & =|\Theta(0)|\|C g\| \\
& =|\Theta(0)|\|g\| \\
& =|\Theta(0)|\|z g\| \\
& =|\Theta(0)|\|\varphi\| \\
& <\|\varphi\| \\
& =\left\|A_{\varphi} K_{0}\right\| .
\end{aligned}
$$

( $C$ is isometric)

(since $|\Theta(0)|<1$ )

\section{REFERENCES}

1. H. Bercovici, Operator theory and arithmetic in $H^{\infty}$, Mathematical Surveys and Monographs, vol. 26, American Mathematical Society, Providence, RI, 1988.

2. N. Chevrot, E. Fricain, and D. Timotin, The characteristic function of a complex symmetric contraction, Proc. Amer. Math. Soc. 135 (2007), no. 9, 2877-2886 (electronic).

3. J. A. Cima, A. L. Matheson, and W. T. Ross, The Cauchy transform, Mathematical Surveys and Monographs, vol. 125, American Mathematical Society, Providence, RI, 2006.

4. J. A. Cima and W. T. Ross, The backward shift on the Hardy space, Mathematical Surveys and Monographs, vol. 79, American Mathematical Society, Providence, RI, 2000.

5. D. N. Clark, One dimensional perturbations of restricted shifts, J. Analyse Math. 25 (1972), 169-191.

6. J. B. Conway, A course in operator theory, Graduate Studies in Mathematics, vol. 21, American Mathematical Society, Providence, RI, 2000.

7. R. B. Crofoot, Multipliers between invariant subspaces of the backward shift, Pacific J. Math. 166 (1994), no. 2, 225-246.

8. N. Dunford and J. T. Schwartz, Linear operators. Part I, Wiley Classics Library, John Wiley \& Sons Inc., New York, 1988, General theory, With the assistance of William G. Bade and Robert G. Bartle, Reprint of the 1958 original, A Wiley-Interscience Publication.

9. P. L. Duren, Theory of $H^{p}$ spaces, Academic Press, New York, 1970.

10. S. R. Garcia, Conjugation and Clark operators, Recent advances in operator-related function theory, Contemp. Math., vol. 393, Amer. Math. Soc., Providence, RI, 2006, pp. 67-111.

11. S. R. Garcia and M. Putinar, Complex symmetric operators and applications, Trans. Amer. Math. Soc. 358 (2006), no. 3, 1285-1315 (electronic).

12. Complex symmetric operators and applications. II, Trans. Amer. Math. Soc. 359 (2007), no. 8, 3913-3931 (electronic).

13. J. Garnett, Bounded analytic functions, first ed., Graduate Texts in Mathematics, vol. 236, Springer, New York, 2007.

14. P. R. Halmos and J. von Neumann, Operator methods in classical mechanics. II, Ann. of Math. (2) 43 (1942), 332-350. 
15. G. Heinig, Not every matrix is similar to a Toeplitz matrix, Proceedings of the Eighth Conference of the International Linear Algebra Society (Barcelona, 1999), vol. 332/334, 2001, pp. 519-531.

16. D. Mackey, N. Mackey, and S. Petrovic, Is every matrix similar to a Toeplitz matrix?, Linear Algebra Appl. 297 (1999), no. 1-3, 87-105.

17. A. L. Matheson and W. T. Ross, An observation about Frostman shifts, Comput. Methods Funct. Theory 7 (2007), no. 1, 111-126.

18. N. Nikolski, Operators, functions, and systems: an easy reading. Vol. 2, Mathematical Surveys and Monographs, vol. 93, American Mathematical Society, Providence, RI, 2002, Model operators and systems, Translated from the French by Andreas Hartmann and revised by the author.

19. A. Poltoratski and D. Sarason, Aleksandrov-Clark measures, Recent advances in operatorrelated function theory, Contemp. Math., vol. 393, Amer. Math. Soc., Providence, RI, 2006, pp. $1-14$.

20. W. T. Ross, Indestructible Blaschke products, Cont. Math. 454 (2008), 119 - 134.

21. D. Sarason, A remark on the Volterra operator, J. Math. Anal. Appl. 12 (1965), 244-246.

22. _ Sub-Hardy Hilbert spaces in the unit disk, University of Arkansas Lecture Notes in the Mathematical Sciences, 10, John Wiley \& Sons Inc., New York, 1994, A Wiley-Interscience Publication.

23. - Algebraic properties of truncated Toeplitz operators, Oper. Matrices 1 (2007), no. 4, 491-526.

24. _ Unbounded operators commuting with restricted backward shifts, Oper. Matrices 2 (2008), no. 4, 583-601.

25. U Unbounded Toeplitz operators, Integral Equations Operator Theory 61 (2008), no. 2, 281-298.

26. J. E. Tener, Unitary equivalence to a complex symmetric matrix: an algorithm, J. Math. Anal. Appl. 341 (2008), no. 1, 640-648.

Department of Mathematics, University of North Carolina, Chapel Hill, North CarOLINA 27599

E-mail address: cima@email.unc.edu

Department of Mathematics, Pomona College, Claremont, California 91711

E-mail address: Stephan.Garcia@pomona.edu

Department of Mathematics and Computer Science, University of Richmond, RichMOND, VIRGINIA 23173

E-mail address: wross@richmond.edu

Department of Mathematics, University of North Carolina, Chapel Hill, North CarOLINA 27599

E-mail address: wrw@email.unc.edu 